\title{
Los baluartes del palacio señorial de Betxí ¿Una fortificación real o ficticia?
} Federico Iborra Bernad $^{\mathrm{a}}, \mathrm{M}^{\mathrm{a}}$ Amparo Sebastiá Esteve ${ }^{\mathrm{b}}$ y Gustavo Aguilella Arzo ${ }^{\mathrm{c}}$

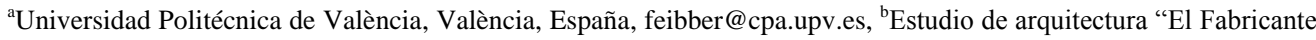
de Espheras”, Valencia, España, oficina@elfabricantedeespheras.com, 'Diputación de Castellón, Castellón, España, gustauaguilella@dipcas.es

\begin{abstract}
The manor house of Betxí, currently in the process of recovery, is one of the forgotten jewels of the Valencian Renaissance. Leisure residence of the powerful family of Cardona, admirals of Aragon, it would be remodeled in the mid-sixteenth century by incorporating an unfinished Renaissance courtyard and four small bastions, very damaged nowadays. Curiously, although the plant design is correct, miniature bastions seem to be too low to serve as defense, and it is still unclear their connection to the building. Somehow it recalls the model of the Villa Farnese in Caprarola, where the bastions have lost their function. Therefore the question arises: Are we facing a real fortification or a local reinterpretation of one of the masterpieces of Italian Renaissance architecture?
\end{abstract}

Keywords: Betxí, Bastion, Joan de Ambuesa, Vignola, Villa Farnese

\section{Introducción}

El palacio de Betxí es probablemente una de las residencias señoriales más singulares de la región valenciana. Ubicado en una pequeña población de la localidad de Castellón, actualmente se encuentra muy alterado y está siendo objeto de un lento proceso de restauración, en el que cabe destacar la reciente actuación sobre el patio, reconocida con un primer premio Ascer en su edición de 2014.

A propósito del edificio ha habido varios autores que han hecho contribuciones de importancia, que han permitido ir desvelando su historia. Sin ánimo de ser exhaustivos, es de justicia recordar El palacio-castillo de Bechí (Traver, 1961), En Pascual Meneu i Meneu (Albiac y Mesado, 2003-2005) y El somni de Pascual Meneu: El Palau de Betxí (Mesado y Gimeno, 2003-2005), D. Pascual Meneu i Meneu. Un «Betxinenc» ilustre (Mesado Oliver, 2003-2005). El PalauCastell de Betxí. L'inventari d'En Sanç Roís de
Liori, vescomte de Galiano (Mesado y Nebot, 2010); El palacio de Betxí. Historia de su construcción a través de la lectura del edificio (Palaia y Tormo, 2009). Merecen aparte ser reseñados algunos informes inéditos, como: Memoria del Plan especial del Palau Castell, (Llop Vidal, Grande Grande y Juan Vidal 2003), Memoria de las Intervenciones Arqueológicas del Palau-Castell de Betxí, (Tamborero y Martínez 2008). A pesar de todo, aún puede ofrecernos nuevas sorpresas.

\section{El palacio fortificado de Betxí}

El palacio es un edificio de planta baja y dos alturas en su fachada principal, con planta cuadrangular de unos 30 metros de lado. Exteriormente se encuentra muy alterado, tras la reedificación de una parte de la fachada en 1903 y el resto en 1969, quedando intacta únicamente la zona central. 
En las cuatro esquinas contó con pequeños baluartes, dos de los cuales se conservan muy alterados hasta la altura del entresuelo, mientras que de los otros apenas quedan las cimentaciones.

Posee además dos elementos significativos de época renacentista, la portada principal y el patio central. La portada se resuelve con un arco de medio punto y sillares almohadillados, mientras que el patio central cuenta con arcadas en la planta baja soportadas por columnas jónicas y arcos de piedra. Sobre éstas se construyó en 1927 una galería en ladrillo, imitando la composición del nivel inferior.

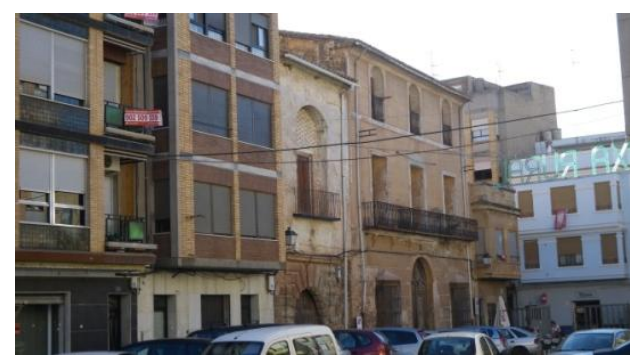

Fig. 1.- El palacio antes de la intervención

La fábrica actual está levantada sobre una fortificación anterior, probablemente del siglo XIV, de la que se han excavado parte de los cimientos (Tamborero, 2008). El edificio podría haberse remodelado o reedificado a mediados del siglo XV, en tiempos de Sancho Ruiz de Liori, hijo del Vizconde de Gallano, quien hacia 1455 vendió sus posesiones italianas y se trasladó a Valencia, donde controlaría las baronías de Betxí y Ribarroja, además de los señoríos de la villa de Gorga y los Valles de Seta y Travadell. El inventario de sus bienes, realizado en 1498, nos da idea de que el edificio medieval tendría dimensiones muy similares a las del actual (Mesado y Nebot, 2010).

De esta época deben ser los muros principales, realizados con un hormigón ciclópeo de cal y grandes cantos procedentes del río, ejecutado con ayuda de un encofrado de tapiales de madera. Presenta arcos apuntados de ladrillo en el patio y, en la última campaña arqueológica, se han encontrado también los restos de una escalera exterior de tradición medieval.

\section{El palacio renacentista}

A mediados del siglo XVI el palacio debió sufrir una nueva remodelación, que lo llevaría a su forma definitiva. El impulsor de la misma sería don Sancho de Cardona, almirante de Aragón y primer marqués de Guadalest, quien casó en junio de 1544 con María de Colón y Toledo, hija de don Diego Colón, primer duque de Veragua y Almirante de las Indias, y nieta de Cristóbal Colón.

La dote de la novia se elevaba a la extraordinaria cantidad de 32.000 ducados (672.000 sueldos valencianos), mientras que el esposo aportaba un aumento de 6000 ducados que avalaba con sus bienes, especialmente de las villas de Betxí y Ondara. Sin embargo, gran parte de este capital se disipó porque en 1548 Sancho de Cardona tuvo que hacer frente a las deudas contraídas por sus padres y abuelos, pagando entre 18 y 20.000 ducados (Blasco, 2014).

La portada del palacio y las troneras de los baluartes fueron construidas en 1559 por Jacques de Pomar (Gómez-Ferrer, 2014), lo que nos hace pensar que en la década de 1550 , resueltos los problemas con los acreedores, debió procederse a la reforma de esta residencia, ubicada en el señorío preferido de don Sancho.

María de Colón falleció en 1564 y debió dejar algún capital a su esposo, puesto que en 1567 está documentada la presencia de varios canteros trabajando en el palacio. Sin embargo, en 1568 Sancho de Cardona debía a los duques de Segorbe casi 3.400 libras (68.000 sueldos) y para pagarlas tuvo que ceder los derechos sobre las baronías de Betxí, Guadalest y Confrides entre 1569 y 1571.

Este sería uno de los motivos por los que no se terminó la parte superior del claustro. El otro fue la condena en 1570 por parte de la Inquisición, acusado de dar permiso a sus vasallos moriscos para reconstruir una mezquita derribada. La pena fue de reclusión perpetua en el convento de San Pablo de Cuenca, pero por su avanzada edad (73 años) se le conmutó con la reclusión dentro del término de la ciudad de Valencia, con la prohibición de ir a cualquiera de sus valles y baronías (Blasco, 2014) 
Sancho de Cardona falleció en 1573, heredando los señoríos su hijo Cristóbal de Cardona y Colón, II marqués de Guadalest y Almirante de Aragón, que también heredó de su madre el ducado de Veragua, el marquesado de Jamaica y el título de Almirante de las Indias. Casado con Ana de Centelles, hija del conde de Oliva, residió en Betxí de manera más o menos estable entre 1573 y su muerte en 1583. Sin embargo, las numerosas deudas y continuos pleitos imposibilitaron que se concluyeran las obras (Gómez-Ferrer, 2014)

\section{Los baluartes del palacio}

Como ya se ha comentado, el palacio fortificado de Betxí presentaba cuatro baluartes en sus esquinas, de los que sólo se conservan dos, muy degradados, aunque las recientes excavaciones han confirmado la existencia de otros dos bajo la actual plaza, que ya había documentado Traver.

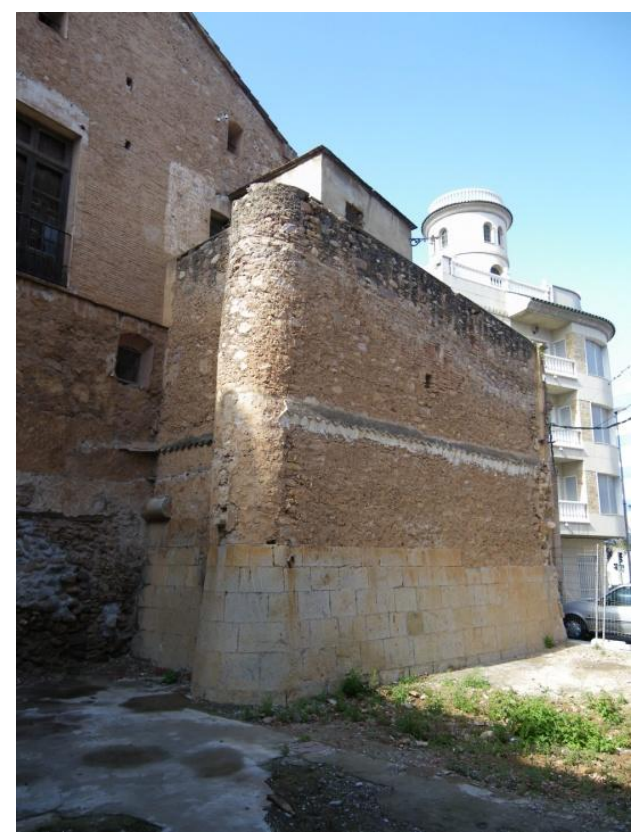

Fig. 2.- Baluarte sudeste del palacio. Puede apreciarse el orejón y, el arranque del bordón.

Según Pascual Meneu, a finales del siglo XIX estaban en pie los cuatro baluartes, que comunicaban con el interior del edificio. Exteriormente eran de sillería, y de argamasa en el interior y la bóveda. En 1903 sólo quedaban dos paredes de las cuatro del baluarte nordeste y, en peor estado, dos caras del sudeste y parte del foso de cuatro metros, cegado al poco tiempo. Los sillares de los baluartes noroeste y sudoeste fueron utilizados para el azud nuevo del río Mijares (Mesado, 2003-2005).

Los restos conservados responden únicamente al cuerpo basamental, aproximadamente hasta la altura del bordón. Su altura ronda los 2,5 metros. La última campaña de excavaciones ha puesto de manifiesto que los baluartes se construyeron dentro del foso primitivo del palacio que, al menos en la zona sur, no se llegó a ampliar para rodearlos.

Resulta muy extraño que los baluartes sean tan bajos, aunque debemos pensar que funcionarían con el apoyo de un foso inundado de agua procedente de una acequia próxima. Autores como Vicente Traver (1961) interpretaron que se trataba de torres, pero tras el estudio de Llop, Grande y Juan, al contar con un levantamiento más preciso, se entendió que se trataba de una fortificación de tipo renacentista perfectamente canónica, como confirma además la presencia de orejones curvos.

Los pequeños baluartes del palacio de Betxí, realizados en la década de 1550 , constituyen un ejemplo pionero en nuestras tierras de la aplicación de las reglas de fortificación moderna. Para entender su importancia podemos compararlo con las cercanas murallas de Mascarell, construidas en 1553-1554 según un modelo de tradición medieval.

Las defensas valencianas más avanzadas de la primera mitad de siglo utilizaron baluartes de planta circular, como en Oliva o Villajoyosa. Este diseño aparece ya en el tratado manuscrito de Francesco di Giorgio (1492) y lo había utilizado de manera muy temprana Ramiro López en la plaza de Salces (1497-1503), aunque en Italia se abandonó a principios del XVI.

Siguiendo este esquema, en 1543 Joan de Cervelló construyó una torre en el nuevo lienzo de la muralla de Valencia, en la zona de la Puerta del Mar. Al año siguiente Felipe II envió al maestre de campo Pedro de Guevara, quien diseñó un complejo sistema de fortificación para 
la ciudad mediante modernos baluartes triangulares, que se levantarían con terraplenes de tierra consolidados mediante fajinas. No será hasta mucho después, en 1574, cuando Gaspar Gregori edificará el bastión de la llamada Casa de Armas, origen de la Ciudadela borbónica (Lillo, 2012).

Llama la atención el gran parecido entre los orejones curvos de la Casa de Armas y del palacio de Betxí. Se trata de una solución de origen italiano que, por ejemplo, no emplearían los ingenieros que trabajaron en área valenciana a mediados del XVI, como Guevara, Antonelli o Gonzaga. No obstante, autores tardíos como Lorini (1596) defienden la idoneidad de los orejones curvos frente a los poligonales, lo que explica la gran aceptación que tendrán en el siglo XVII.

La presencia de estos orejones curvos en los baluartes de la Casa de Armas de Valencia y Betxí establece un importante nexo de unión entre ambas obras. Por tanto, no sería descabellado atribuir la autoría de Betxí también a Gregori, quien en la segunda mitad del XVI era considerado un reputado diseñador de arquitectura. Suyos eran, por ejemplo, los proyectos para los dos cruceros del Hospital General de Valencia (1546 y 1588), la Obra Nova de la Catedral (1566), la parte superior del torreón de la Generalidad (1567), la desaparecida sacristía de San Martín (15711573) y una propuesta no realizada para la iglesia del Salvador de Cocentaina (1576). También podría estar detrás del proyecto para la iglesia del colegio del Patriarca (1590) en cuyas capitulaciones se le nombra como perito para reconocer la piedra (Gómez-Ferrer, 1995).

Los orejones curvos están presentes en las primeras obras impresas sobre fortificaciones. Aparecen alternando con los rectos en algunas láminas del libro de Cataneo, siendo omnipresentes en el tratado de Zanchi, en este caso vinculados a traveses cóncavos. Ambos se publicaron en Venecia en 1554, y constituían el tipo de bibliografía accesible a cualquier diletante. Sin embargo, la definición de detalle es insuficiente para alguien que no esté familiarizado con este tipo de estructuras.
Una segunda posibilidad es la presencia de alguien que conociera de primera mano estructuras similares. En este sentido, cabe destacar que el tamaño de los orejones en los dos ejemplos valencianos, con un diámetro de aproximadamente $1 / 3$ del ancho del baluarte, es muy reducido en comparación con las obras de la época. Lo habitual era darle entre $1 / 2$ y $2 / 3$ del ancho, y arquitectos como Sangallo trabajaron con dimensiones aún mayores a principios del XVI. Sin embargo, encontramos soluciones parecidas a las valencianas en el sur de Italia, principalmente en las fortificaciones realizadas en Sicilia por Antonio Ferramolino en la década de 1530.
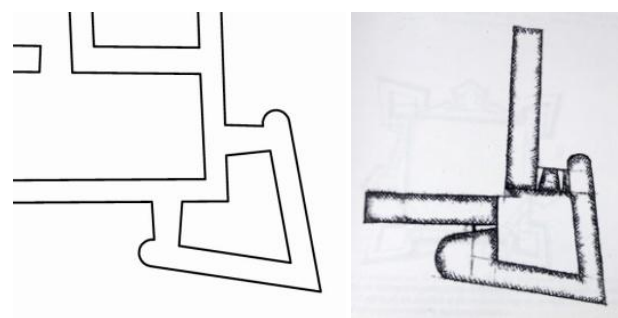

Fig. 3.- Comparación entre uno de los baluartes de Betxí y el recogido por Pedro Luis Escrivá.

Los esquemas de orejones usados por Sangallo y Ferramolino aparecen en una de las ilustraciones de la Apología en escusation de las fábricas del Reyno de Nápoles (1538), redactado por Pedro Luis Escrivá para defenderse de las críticas dirigidas contra su innovador proyecto del Castillo de Sant'Elmo en Nápoles.

Este caballero valenciano fue uno de los principales ingenieros militares de su tiempo. Diseñó varias fortalezas, entre las que destacan las de l'Aquila y el castillo de Sant'Elmo, y escribió Edificio militar, el primer tratado moderno de poliorcética, que nunca fue publicado (Sánchez, 1995).

Resulta tentador plantear una posible relación del propio Escrivá con los baluartes de Betxí, o de alguno de sus parientes que aparecen documentados dirigiendo sus obras. La alternativa es pensar en un ingeniero del círculo de Ferramolino o, simplemente, alguien familiarizado con las modernas realizaciones militares en el sur de Italia. 


\section{La portada renacentista del palacio}

Aunque no se trate de un elemento defensivo propiamente dicho, la portada del palacio de Betxí puede tener importancia a la hora de comprender el tipo de actuación que se estaba llevando a cabo en el señorío de los Cardona.

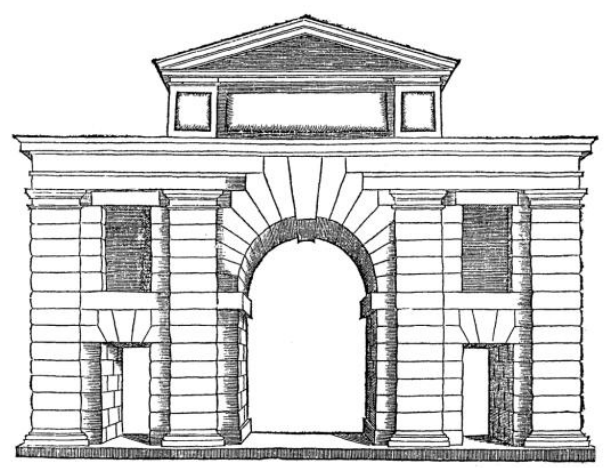

Fig. 4.- El orden rústico en Serlio (L. IV, VIIIv)

Esta portada responde a lo que se ha venido a llamar orden rústico, con un aspecto inacabado que evoca algunas realizaciones romanas de época imperial. El orden rústico viene recogido dentro del Cuarto Libro del tratado de Serlio (1540), cuyas ilustraciones podrían haber guiado a un artista local para la realización de la portada (Bérchez, 1994; Palaia y Tormo, 2009).

Gaspar Gregori y otros constructores de la época emplearon el repertorio de Serlio con soltura a mediados de siglo. Sin embargo, la portada de Betxí presenta singularidades que no están presentes en los modelos de Serlio, como las impostas, capiteles y basas liberadas de almohadillado. Algunas de estas características aparecerían en el dibujo de una puerta de la Villa Farnese de Caprarola que incluyó Vignola en su tratado Regola delli cinque ordini d'architettura (1562).

Sin embargo, hay que tener en cuenta que en Betxí se estaba trabajando en 1559. Además, las similitudes entre nuestra portada y la de la Villa Giulia de Roma, construida por Giacomo Barozzi da Vignola entre 1551 y 1553, son más que sospechosas para poder obviarlas. No es sólo la portada. También la composición de la fachada de la Villa Giulia guarda semejanzas con Betxí. Si obviamos las hornacinas laterales, en el primer piso tenemos cinco huecos, de los cuales el central queda incorporado dentro de un gran vano arqueado, prácticamente del mismo tamaño que la puerta.

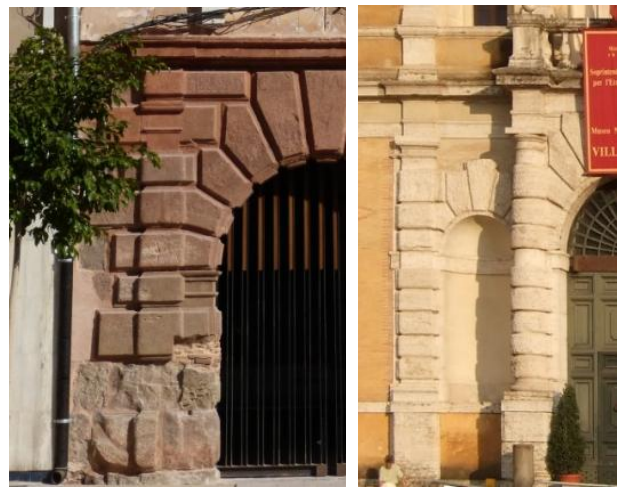

Fig. 5.- Comparativa entre las portadas de Betxí y de la Villa Giulia, en Roma.

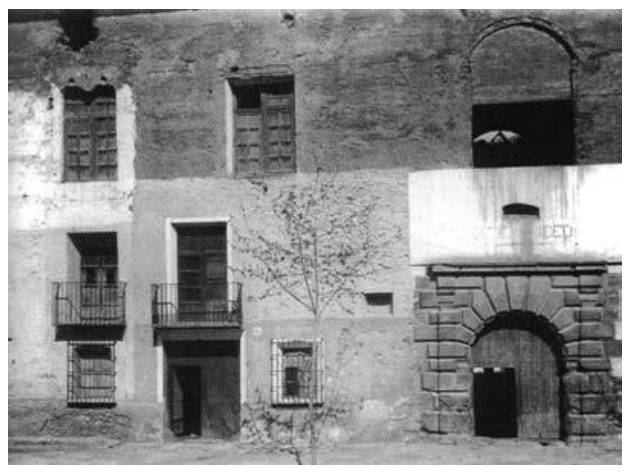

Fig. 6.- Fachada del palacio de Betxí antes de su derribo parcial en 1969.

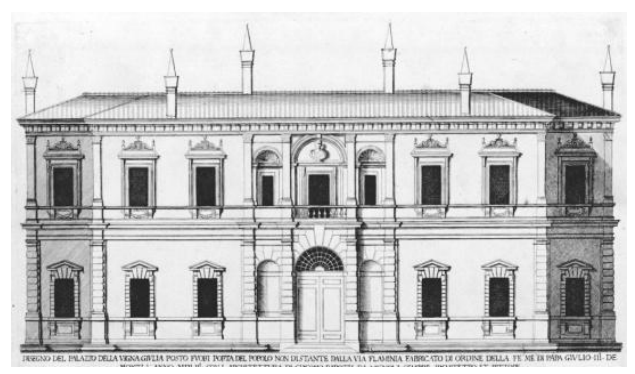

Fig. 7.- Villa Giulia, Roma (1551-1553).

Otra de las incógnitas del palacio de Betxí es la enorme distancia entre el entablamento de la portada y el arranque del hueco superior. Esta separación se debe a la proporción de la portada, 
en relación de $1 / 5$ a 1 . En Villa Giulia esta relación es de 2 a 1 porque la portada apoya sobre pedestales, quizá suprimidos en Betxí para adaptarse a la altura de un hueco preexistente o a la tradición local.

¿Qué tiene que ver todo esto con la arquitectura abaluartada? Realmente poco o nada, pero es importante establecer estos paralelos porque en esta misma época Giacomo Barozzi da Vignola estaba proyectando una de sus obras maestras: la Villa Farnese en Caprarola. Aunque la ejecución no comenzó hasta 1559, fue en 1556 cuando el cardenal Alessandro Farnese le encargó la construcción de este singular palacio suburbano levantado sobre una fortificación abaluartada precedente.

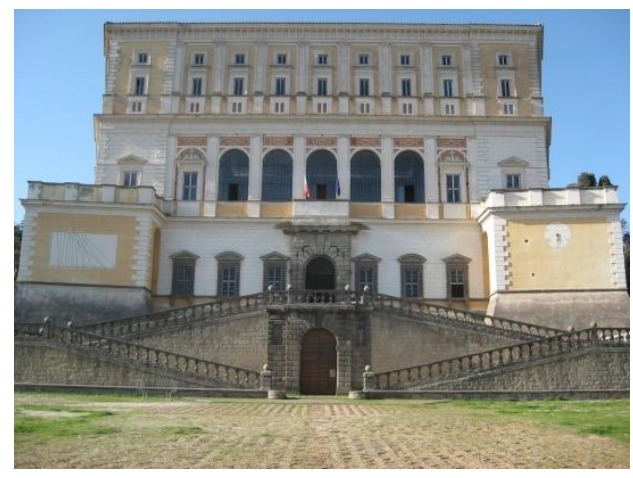

Fig. 8.- Villa Farnese, Caprarola (1559-1575).

En la Villa Farnese también tenemos dos huecos del mismo tamaño superpuestos uno encima del otro, aunque en este caso son dos puertas situadas en planos diferentes. Si tomáramos un plano de alzado frontal, comprobaríamos que ambas quedan bastante separadas, como ocurre en Betxí, ya que sobre la primera existe un tramo de escalera a modo de puente que sirve para llegar a la segunda y acceder al edificio. Con este recurso Vignola corregía magistralmente la percepción del visitante, que cree ver un balcón ubicado directamente sobre la puerta inferior.

Toda la parte comentada de edificio, flanqueada por los baluartes de la fortificación, actúa como basamento de tres plantas más, en las que destaca la principal con su gran loggia abierta. En Betxí también hubo una "loggia” o, más bien, un porche como los que son habituales rematando la fachada de los palacios valencianos, cuya existencia ha sido comprobada por el profesor Santiago Tormo a partir de la fotografía térmica de la zona transformada a principios del siglo $\mathrm{XX}$.

\section{Restitución hipotética}

Interpretar cómo fue el exterior del palacio de Betxí es complejo, puesto que no responde a una tipología de fortificación convencional, sino que se trata de una mezcla de fortín y residencia de recreo aristocrática. Sin embargo, conociendo los posibles modelos nos atreveríamos a proponer una solución, matizando algunas cuestiones.

Conocemos la composición de la parte izquierda de la fachada principal perdida, a través de fotografías antiguas y del croquis dibujado por Vicente Traver en 1961. A partir de ahí es fácil considerar una simetría, que asumiría algunos de los huecos actuales de la transformación llevada a cabo por Pascual Meneu en la parte derecha.
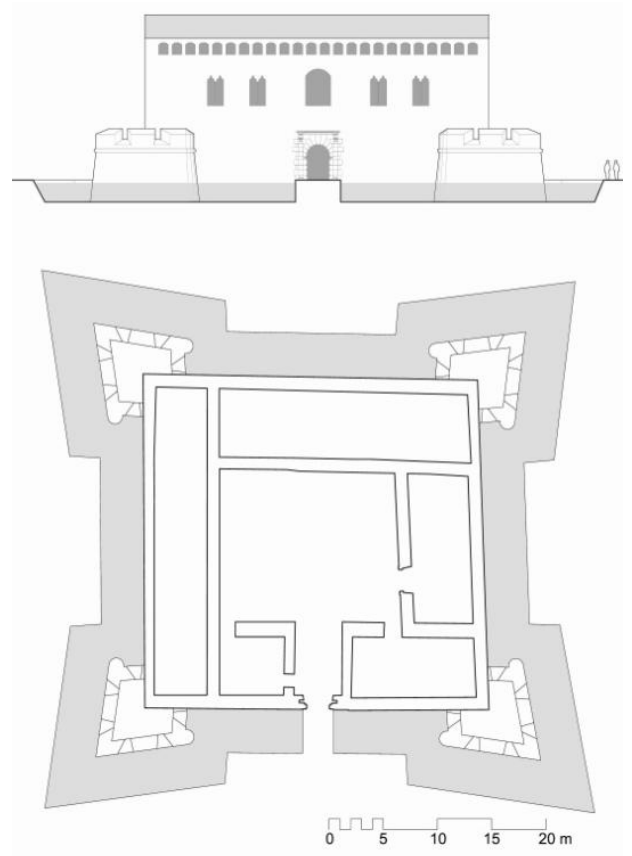

Fig. 9.- Restitución hipotética del palacio

A ambos lados de la fachada tendríamos los dos baluartes desaparecidos, similares a los que se 
conservan al otro lado. Aunque no está claro que llegara a ejecutarse el foso, estos baluartes no se entienden sin un elemento de separación que evite un asalto directo.

Existiendo un foso, relleno de agua gracias a una acequia que pasa por las proximidades del palacio, podemos considerar que sobre el bordón podría venir ya un antepecho, y que las plataformas defensivas serían accesibles desde los entresuelos del palacio, de modo que el sistema defensivo tendría autonomía respecto a la residencia. Sabemos también que en 1559 se construyeron 24 troneras, doce lisas y doce almohadilladas, que corresponderían con toda seguridad a los antepechos de los cuatro baluartes (Gómez-Ferrer, 2014).

Estos baluartes bajos, ubicados a los pies de una estructura anterior, nos recuerdan a las defensas proyectadas por Guevara para la ciudad de Valencia una década antes, o a la doble línea del Castelnuovo de Nápoles. En el fondo funcionarían de manera parecida a una barbacana medieval, pero conectados al cuerpo principal del edificio.

Se puede pensar en otras soluciones alternativas, que en principio descartamos. Una sería la de elevar los baluartes hasta el piso principal, como propone Traver en su reconstrucción, probablemente inspirado en el volumen actual del baluarte sudeste, parcialmente demolido y después reedificado con mampostería. El aspecto sería parecido al de la Villa Farnese, aunque allí el segundo cuerpo de sus baluartes parece un añadido de Vignola.

Otra posibilidad sería la de alcanzar el nivel de cubierta, como en el castillo de l'Aquila, contando con una terraza de maniobra. En este caso, lo que prima es la posibilidad de poder atacar con cañones a un enemigo lejano, como ocurre en el caso de la iglesia fortificada de Vinaroz y algunas torres de costa. No parece el caso del palacio de Betxí, población ubicada a más de 10 kilómetros de la costa, donde el sistema defensivo estaría pensado en todo caso para repeler incursiones berberiscas o revueltas de los pobladores moriscos.

\section{Conclusiones}

El análisis y comprensión del palacio de Betxí sugiere que la intervención del siglo XVI podría ir más allá de una simple fortificación de costa. La vieja residencia señorial medieval, con sus desmesurados muros, probablemente era suficiente para las necesidades reales de defensa.

En nuestra opinión, lo que hicieron los Cardona en Betxí fue dejarse seducir por la idea de una villa de recreo construida sobre un moderno fortín renacentista, como la que Vignola estaba preparando a las afueras de Roma para el cardenal Alessandro Farnese. Para el proyecto tuvieron que llegar algunos dibujos directamente de Italia, única posibilidad de explicar las semejanzas entre la portada principal valenciana y la de la Villa Giulia, también de Vignola.

Podríamos considerar la posibilidad de que el cantero Jacques de Pomar, de origen francés y documentado por primera vez en Betxí (GómezFerrer, 2014) hubiera estado antes en Italia y fuera autor de las trazas. Sin embargo, al comparar ambas portadas resulta evidente, por detalles como el tratamiento del intradós o la hilada de la imposta, que se partió de un dibujo. Más difícil es, sin embargo, establecer si lo que se recibió fue un proyecto completo o simplemente esquemas de lo que Vignola estaba trabajando en ese momento.

Hubo una adaptación local de la propuesta, en la que debió intervenir alguien conocedor de las fortificaciones realizadas por los virreyes españoles en las tierras de Nápoles y Sicilia. La geometría de los baluartes de Betxí es perfectamente canónica, con orejones reducidos, $\mathrm{y}$ no tenemos constancia de precedentes en tierras valencianas con estas características, después retomadas en la Casa de Armas.

Las necesidades defensivas no debieron ser el principal objetivo, puesto que en la época se estaban resolviendo torres o murallas de costa con medios mucho más primitivos. Tendríamos, por tanto, un intento de emular, dentro de las posibilidades locales, el fantástico proyecto de la Villa Farnese de Caprarola, en un momento en que las modernas fortificaciones abaluartadas constituían toda una novedad en España. 


\section{Referencias}

Albiac i Mesado, V. (2003-2005). "Pascual Meneu i Meneu”. Estudis Castellonencs. 10. pp. 735-740.

Bérchez, J. Jarque, F. (1994). Arquitectura renacentista Valenciana, 1500-1570. Ed. Bancaja. Valencia.

Blasco Cobeño, J. F. (2014) "El palau”. La taberna. XXXVI. http://lataberna.eu/index.php/historialocal. consultado en abril de 2015.

Cataneo, P. (1554). I quattro primi libri di Architettura di Pietro Cataneo Senese... Ed. Figliuoli di Aldo. Venecia.

Gómez-Ferrer Lozano, M. (1995). "Una traza renacentista del arquitecto valenciano Gaspar Gregori”. Saitabi. 45. pp. 223-232.

Gómez-Ferrer Lozano, M. (1998). Arquitectura en la Valencia del siglo XVI. El Hospital General y sus artífices. Ed. Albatros. Valencia.

Gómez-Ferrer Lozano, M. (2014). "El palacio renacentista de Betxí (Castellón). Aportaciones a su historia constructiva". Artigrama. 29. (en prensa).

Lillo Giner, S., (2012). La Ciudadela de Valencia. Origen, evolución y análisis gráfico. Tesis Doctoral. Universidad Politécnica. Valencia.

Llop, E., Grande, F., Juan F. (2003). Memoria del Plan especial del Palau Castell.

Lorini, B. (1596). Delle fortificationi di buonaiuto lorini ... libri cinque. Ed. Gio. Antonio Rampazetto. Venecia.

Mesado i Gimeno, X., Nebot i Garcia, F. (2010). El Palau-Castell de Betxí. L'inventari d'En Sanç Roís de Liori, Vescomte de Gallano. Ed. Associació cultural d'amics del Palau de Betxí - Diputació de Castelló. Betxí.

Mesado i Ximeno, X., (2003-2005). "El somni de Pascual Meneu: El Palau de Betxí”. Estudis Castellonencs. 10. pp. 741-748.

Mesado Oliver, N. (2003-2005). "D. Pascual Meneu i Meneu. Un 'Betxinenc' ilustre”. Estudis Castellonencs. 10. pp. 749-880.

Palaia, L., Tormo, S. (2008). Informe final. Estudio constructivo. Palacio de los condes de Ariza. Betxí, Castellón.

Palaia, L., Tormo, S. (2009). "El palacio de Betxí. Historia de su construcción a través de la lectura del edificio". Actas del Sexto Congreso Nacional de Historia de la Construcción. Ed. Instituto Juan de Herrera. Madrid. pp. 1019-1028.

Sánchez Gijón, A. (1995). Pedro Luis Escrivá, caballero Valenciano, constructor de Castillos. Ed. Ajuntament de València. Valencia.

Serlio, S. [1552]. (1986). De todas las obras de arquitectura y perspectiva de Sebastian Serlio de Bolonia. Edición fac-simil. Ed. Colegio oficial de aparejadores y arquitectos técnicos de Asturias. Oviedo.

Tamborero Capilla, L., Martínez Porral R. (2008). Memoria de las Intervenciones Arqueológicas del Palau-Castell de Betxí, ( $2^{a}$ Fase).

Traver, V., (1961). "El palacio-castillo de Bechí", Boletín de la Sociedad Castellonense de Cultura, 37 IV, pp. 253-67.

Zanchi, G. B. (1554). Del modo di fortificar la cittá. Ed. Plinio Pietrasanta. Venecia. 